\title{
Multiplex Capillary Denaturing High- Performance Liquid Chromatography with Laser-Induced Fluorescence Detection
}

BioTechniques 30:1332-1338 (June 2001)

\author{
W. Xiao, D. Stern ${ }^{1}$, M. Jain, \\ C.G. Huber ${ }^{2}$, and P.J. Oefner \\ Stanford University, Palo Alto, \\ CA, ${ }^{1}$ Affymetrix, Santa Clara, \\ CA, USA, and ${ }^{2}$ Leopold- \\ Franzens-University, \\ Innsbruck, Austria
}

\begin{abstract}
Denaturing high-performance liquid chromatography (DHPLC) is a sensitive, robust, and operationally inexpensive method for the detection of single-base substitutions and small deletions and insertions. To increase sample throughput, we have developed a multiplexing strategy using fluorophores to distinguish different PCR products. The system combines recent advances in the synthesis of monolithic poly(styrene-divinylbenzene) capillary columns with four-color confocal argon ion laser-induced fluorescence detection. Depending on the change in retention caused by the fluorophores, adjustments in the analysis temperature may be required to ensure the maximum mutation detection sensitivity.
\end{abstract}

\section{INTRODUCTION}

Because of its high sensitivity $(>96 \%)$ and specificity (>99\%) in the detection of mutations in DNA fragments as large as $1000 \mathrm{bp}(2,7,9,14$, 20,24), denaturing high-performance liquid chromatography (DHPLC) is widely used in the analysis of DNA sequence variation (http://insertion. stanford.edu/pub.html). Applications of DHPLC include, among others, the discovery of simple sequence polymorphisms $(1,23)$, the mapping of genes $(19,21)$, the mutational analysis of candidate genes $(3,17,24)$, and the targeted screening for induced mutations (12).
Mutational analysis with partially denaturing HPLC is based on the prinates double-stranded heteroduplex species with different conformational structure and melting properties from perfectly matched homoduplexes. Under conditions of partial denaturation, this gives rise to different retention of the double-stranded heteroduplexes and homoduplexes using ion-pair reversedphase HPLC (IP-RP-HPLC), with heteroduplex species typically eluting before the homoduplex species (16). Ideally, as many as four peaks appear in the chromatogram, representing each of the four hybridized species. ciple that mismatched base pairing cre-

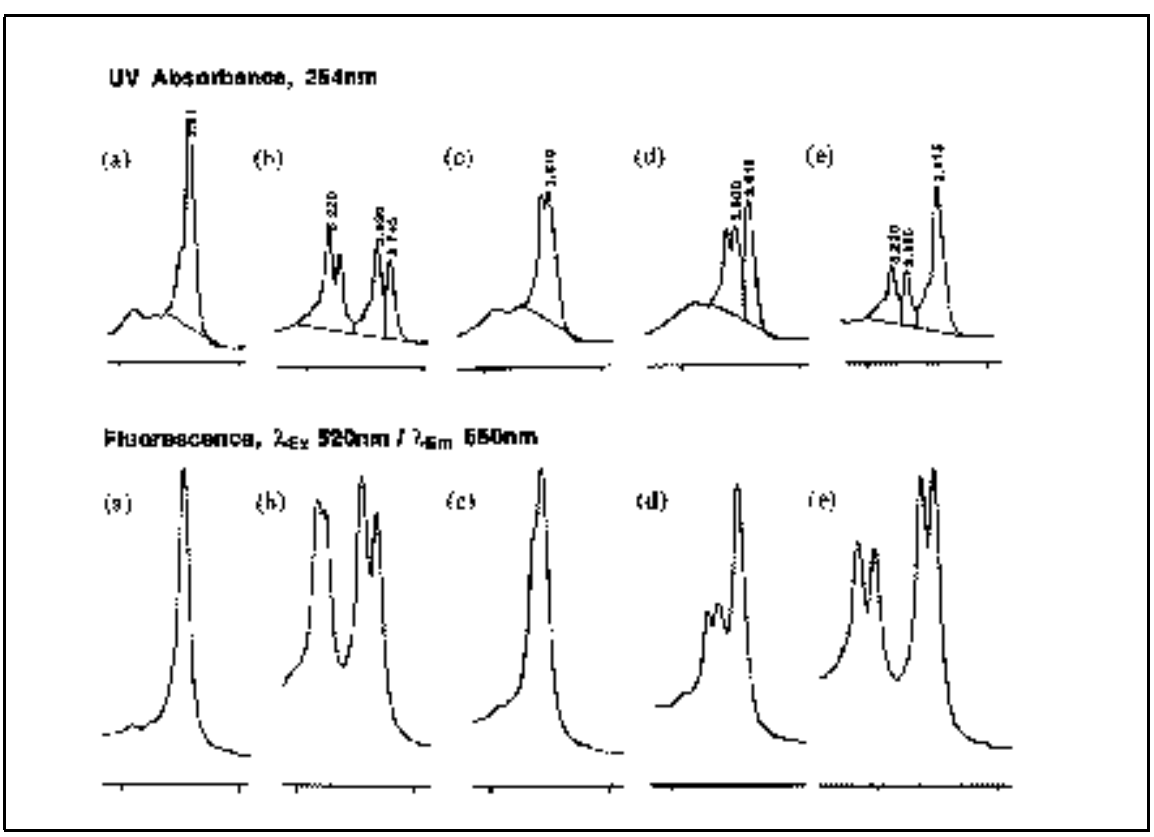

Figure 1. Separation of unlabeled and fluorescent dye-labeled (HEX) 393-bp homoduplex and heteroduplex species. Chromatograms show the analyses of a monomorphic control (a) and four different mismatches (b-e) located at nucleotide positions 73 (TC), 161 (GA), 246 (CT), and 289 (TC), respectively. DH PLC was carried out at $56^{\circ} \mathrm{C}$, with a $50 \times 4.6 \mathrm{~mm}$ i.d. stainless steel column packed with nonporous $2-\mu \mathrm{m}$ PS/DVB-C18 particles, by increasing the relative concentration of buffer B from 50\% to 55\% in $0.5 \mathrm{~min}$ and from 55\% to $64.9 \%$ in $5 \mathrm{~min}$. The UV absorbance detection was performed at $254 \mathrm{~nm}$, while the excitation and the emission wavelengths for fluorescence detection were set at 520 and $550 \mathrm{~nm}$, respectively. 
As various genome projects that aim at large-scale discovery and the genotyping of single-nucleotide polymorphisms (SNPs) are well underway, there are compelling needs to further develop DHPLC into a high-throughput technique for both the discovery and the genotyping of SNPs $(12,21)$. Recently, it has been demonstrated that using monolithic capillary columns synthesized in situ by copolymerization of styrene and divinylbenzene yields high resolving power of ss and dsDNA, which compares well to the resolving power obtained previously with micropellicular poly(styrene-divinylbenzene) (PS/DVB) (18). As a consequence of improved concentration sensitivity offered by the capillary format, it is now possible to combine DHPLC with laserinduced fluorescence detection similar to capillary electrophoresis in DNA se- quencing $(5,10,22)$. The present study demonstrates the successful, multiplex capillary DHPLC analysis of PCR products labeled with fluorophores of four different colors. It also describes the impact of various fluorophores on retention and melting behavior.

\section{MATERIALS AND METHODS}

\section{DNA Sequence and Dye-Labeled Primers}

We studied the following two human Y-chromosome sequences containing four and one SNP, respectively. The first one was $393 \mathrm{bp}$ and was amplified with the following forward and reverse primers: 5'-TGGTAAACTCTACTTAG TTGCCTTT- $3^{\prime}$ and $5^{\prime}$-CAGCGAATTA GATTTTCTTGC-3'. The forward pri- mer was labeled with HEX. The four mismatches were located at nucleotide positions 73 (TC), 161 (GA), 246 (CT), and 289 (TC), respectively, from the $5^{\prime}$ end of the product. The second product was $209 \mathrm{bp}$, and the following forward and reverse primers were used: $5^{\prime}$-AGG CACTGGTCAGAATGAAG- $3^{\prime}$ and 5'AATGGAAAATCAGCAGCTCCCC$3^{\prime}$. The single mismatch was located at nucleotide position 168 (AG). The forward primer was labeled with different fluorophores. Oligonucleotides labeled with HEX [emission maximum (12), $555 \mathrm{~nm}$ ], TET (545 nm), and NED (580 $\mathrm{nm})$, were obtained from Applied Biosystems (Foster City, CA, USA). Unlabeled primers labeled with 6-Fam (520 $\mathrm{nm})$, TAMRA $(580 \mathrm{~nm}), \operatorname{ROX}(605 \mathrm{~nm})$, BODIPY FL (515 nm), and BODIPY R6G (550 nm) were obtained from Life Technologies (Rockville, MD, USA).

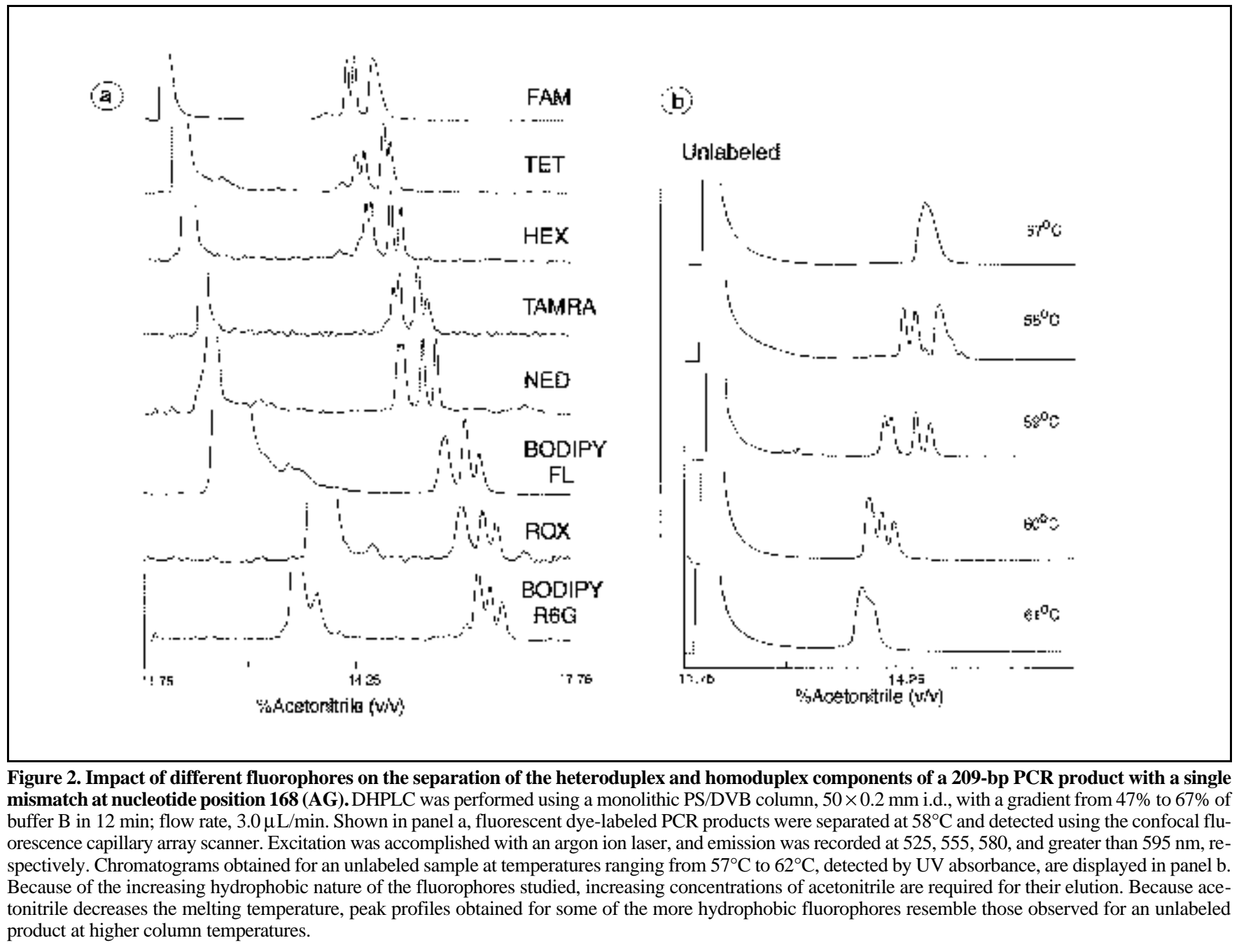




\section{Research Report}

\section{PCR}

Each allele was amplified separately in a $50-\mu \mathrm{L}$ PCR that contained $1 \mathrm{U}$ AmpliTaq Gold ${ }^{\circledR}$ DNA polymerase (Applied Biosystems), $10 \mathrm{mM}$ Tris$\mathrm{HCl}, \mathrm{pH} 8.3,50 \mathrm{mM} \mathrm{KCl}, 2.5 \mathrm{mM}$ $\mathrm{MgCl}_{2}, 0.1 \mathrm{mM}$ each of the four dNTPs, $0.2 \mu \mathrm{M}$ each forward/reverse primers, and $50 \mathrm{ng}$ genomic DNA. The thermocycling protocol comprised an initial denaturation at $95^{\circ} \mathrm{C}$ for $10 \mathrm{~min}$ to activate AmpliTaq Gold, 14 cycles of denaturation at $94^{\circ} \mathrm{C}$ for $20 \mathrm{~s}$, primer annealing at $63^{\circ} \mathrm{C}-56^{\circ} \mathrm{C}$ with $0.5^{\circ} \mathrm{C}$ decrements, extension at $72^{\circ} \mathrm{C}$ for 1 min, followed by 20 cycles at $94^{\circ} \mathrm{C}$ for $20 \mathrm{~s}, 56^{\circ} \mathrm{C}$ for $1 \mathrm{~min}, 72^{\circ} \mathrm{C}$ for $1 \mathrm{~min}$, and a final 5 -min extension at $72^{\circ} \mathrm{C}$. PCR yields were determined semiquantitatively on ethidium bromide-stained agarose gels. For DHPLC analysis, unpurified PCR products of each allele were mixed at an equimolar ratio and subjected to a 3 -min $95^{\circ} \mathrm{C}$ denaturing step, followed by gradual reannealing from $95^{\circ} \mathrm{C}$ to $65^{\circ} \mathrm{C}$ over $30 \mathrm{~min}$.

\section{DHPLC}

The capillary HPLC instrument and the monolithic column have been described previously (18). The system consists of a pump (Maxi-Star K-1000; Knauer, Berlin, Germany), a degasser (model DG-1210; Uniflows, Tokyo, Japan), a column oven (Mistral; Varian, Walnut Creek, CA, USA), a four-position microscale injector (model 7410; Rheodyne, Rohnert Park, CA, USA) with a $1-\mu \mathrm{L}$ internal sample loop, a laser-induced fluorescence scanner, and a PC-based data system. A T-piece is used to split the primary flow and deliver gradients to the column.

To test the impact of fluorescent dye on the separation of homo- and heteroduplex species, a column packed with alkylated, nonporous 2- $\mu \mathrm{m}$ PS/ DVB particles (6) was used on a ProStar Helix HPLC system (Varian) equipped with a spectrofluorometer (model FP920; Jasco, Easton, MD, USA).

The composition of the buffers is (A) 100 mM TEAA, $\mathrm{pH} 7.0,0.1 \mathrm{mM} \mathrm{Na}_{4}$ EDTA and (B) 100 mM TEAA, pH 7.0, $0.1 \mathrm{mM} \mathrm{Na} 4$ EDTA, $25 \%$ acetonitrile. The gradient profiles used for the separations are given in the figure legends.

\section{Laser-Induced Fluorescence Detector}

The confocal fluorescence capillary array detector was constructed according to the original scanner by Kheterpa et al. (8), designed for the purpose of capillary array electrophoresis-based DNA sequencing. This scanner collects four-color fluorescence data up to 25 capillaries in parallel. Briefly, capillaries are mounted onto a translation stage driven 
by a computer-controlled, microstepping indexer (Zeta6104; Parker Hannifin, Cleveland, OH, USA). An excitation beam $(488 \mathrm{~nm})$ from an argon ion laser is focused into the capillary array through a microscope objective. Fluorescence from the capillaries is collected by the same objective and fractioned successively by four longpass dichroic beam splitters (Omega Optical, Brattleboro, VT, USA) with transmission wavelengths $(>50 \% \mathrm{~T})$ of $505,540,570$, and $595 \mathrm{~nm}$, respectively. Bandpass filters $\left(\mathrm{OMIGA}^{\mathrm{TM}}\right.$ 525DF30, 555DF30, and 585DF20) are used on the light reflected by each of the first three beam splitters and a 595-nm longpass filter after the last beam splitter. Filtered light in each fraction is then detected by a photomultiplier tube (model HC120-05; Hamamatsu, Bridgewater, NJ, USA). Signals are lowpass filtered by 4-pole Bessel filters (model 824L8L-4; Frequency Devices, Haverhill, MA, USA) and digitized by a 16-bit data acquisition board (CIO-DAS1402/16; Computer Boards, Middleboro, MA, USA). Fourcolor data are filtered using a five-point filter and reduced using color-separation matrices. A computer program synchronizes the movement of the translation stage and data acquisition, allowing each capillary to be scanned twice per second.

\section{RESULTS AND DISCUSSION}

\section{Effect of Fluorescent Dye on Heteroduplex Detection}

High-resolution liquid chromatography of fluorescent dye-labeled nucleic acids has been discussed in detail by Oefner et al. (15). The sensitivity of fluorescence detection was shown to be 30-100-fold higher than that of UV absorbance detection. The measurement of fluorescence from primer-labeled PCR products is therefore an attractive alternative for highly sensitive mutation analysis by DHPLC (4). Similar to the sizing of microsatellite markers (11), fluorescent dye-labeling strategies can be used to increase sample throughput by using different fluorophores as sam ple identifiers. Although multiplexing based on differences in product size has been demonstrated (16), the actual gain in throughput is minimal because of the increased analysis time. Figure 1 shows a comparison of chromatograms obtained for unlabeled and fluorescent dye (HEX)-labeled polymorphic PCR products, detected either by UV absorbance or fluorescence. The chromatographic profiles from fluorescence largely resemble the ones from UV absorbance, which corroborates the feasibility of employing fluorescence-based detection in DHPLC analysis. The slight differences in the peak pattern observed between the chromatograms result mainly 
from the impact of the fluorescent dye on retention that increases because of the hydrophobic nature of the fluorophore. The longer a DNA fragment is retained, the higher the concentration of acetonitrile required for its elution, which in turn causes more extensive denaturation of the product. The changes in the peak pattern are therefore similar to those seen with increasing column temperature (16), which is the paramount factor influencing the detection power of DHPLC. Dyes with higher hydrophobicity bind stronger to the column and are expected to have a larger effect on the chromatographic profiles than more hydrophilic dyes. For the analysis with multiplexed PCR products, attention must therefore be paid to select the group of dyes that share a sim ilar retention behavior to ensure maximum mismatch detection sensitivity.

\section{Laser-Induced Fluorescence Detection with Capillary DHPLC}

The peak concentration of analyte in the effluent of conventional 4.6-mm i.d. columns has proven too low to allow the use of a laser-induced fluorescence detector. With the $200-\mu \mathrm{m}$ i.d. column, peak concentration is now sufficiently high to allow the detection of fluorescent dye-labeled nucleic acids. The four-color confocal fluorescence capillary array scanner with channels centered at 525, 555, 580, and greater than $595 \mathrm{~nm}$ was coupled with capillary HPLC, and different dyes linked to 20-mer oligonucleotides were tested for detection limit and retention time. Using the 488-nm laser excitation, all the fluorescent dyes had comparable emission levels detectable by the scanner (the lowest from ROX is about $50 \%$ of the highest from FAM). The lower detection limit was about 0.5 fmol for the dye-labeled 20-mer oligonucleotides. The affinity to column determined by retention time, is, in increasing order, FAM, TET, HEX, TAMRA, NED, BODIPY FL, ROX, and BODIPY R6G. The impact of the different fluorophores on the chromatographic profile of a 209-bp polymorphic PCR product containing a single mismatch (AG) was determined by running all samples under identical gradient and temperature conditions. A comparison of Figure 2, pan- els a and b, shows that the FAM-labeled PCR product yields a heteroduplex profile virtually identical to that of an unlabeled product. All other fluorophores led to noticeable increases in retention. Chromatographic profiles obtained for TET- and HEX-labeled products were quite similar to that of FAM, but the peak patterns obtained with NED and TAMRA more closely resembled that of an unlabeled product analyzed at $59^{\circ} \mathrm{C}$. The products labeled with ROX and the BODIPY dyes, which were retained the strongest, resembled an unlabeled product analyzed at $60^{\circ} \mathrm{C}$.

Figure 3 shows the multiplex detec- tion of PCR products labeled with four different fluorescent dyes (FAM, HEX, NED, and ROX, respectively) that have been used widely in four-color DNA sequencing. The four products were generated separately and mixed together before injection, separated by the capillary column, and detected by the fourcolor confocal fluorescence capillary array scanner. Species tagged with different dyes are identified by the characteristic emission spectrum of each attached fluorophore measured in the four channels of the detector, as shown in Figure 3, panels a-c. The monomorphic PCR products, yielding single peaks

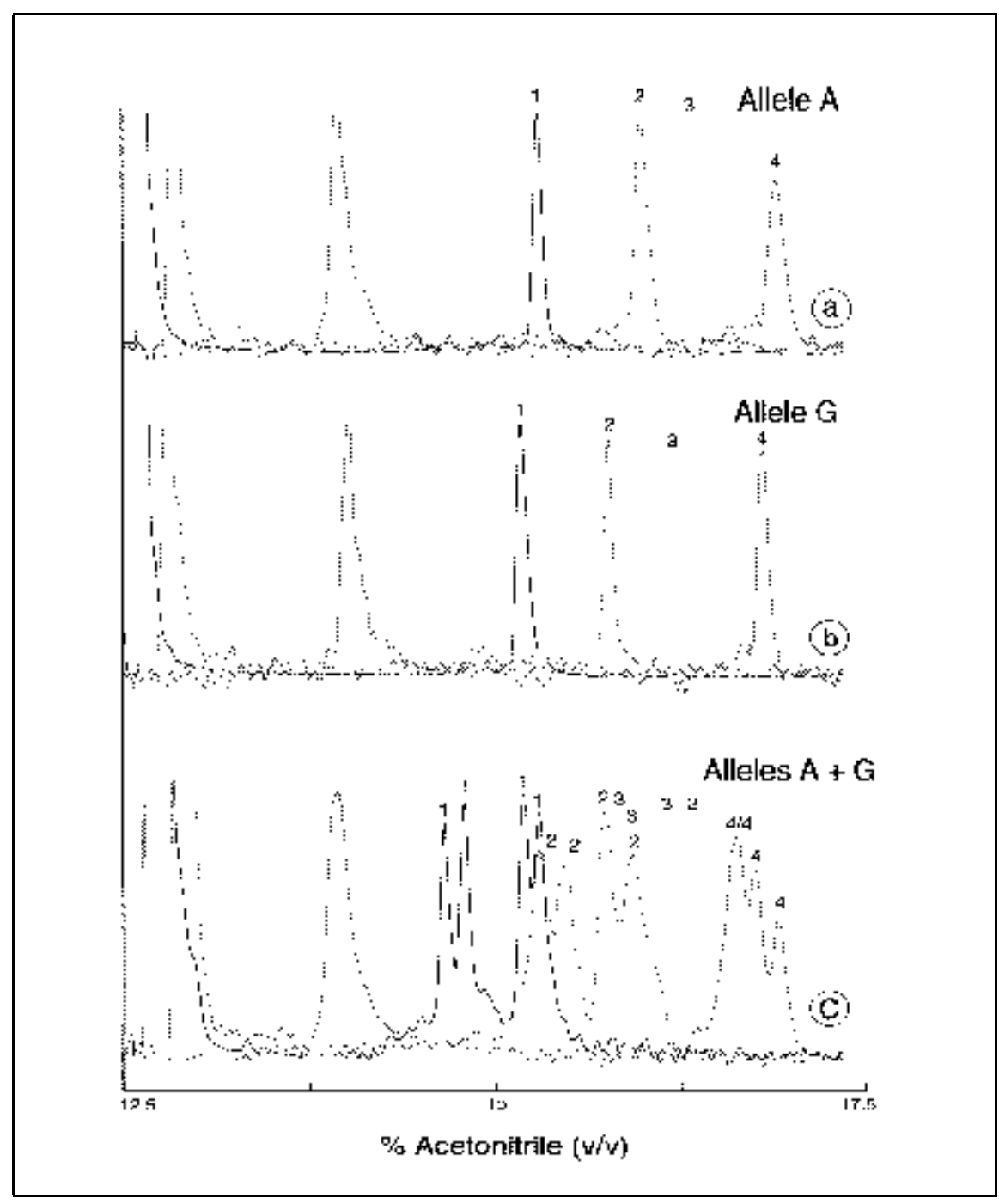

Figure 3. Capillary DHPLC and laser-induced fluorescence detection of a single-base mismatch in a 209-bp product tagged with FAM (peak 1), HEX (peak 2), NED (peak 3), and ROX (peak 4). Color-separated traces are shown in panel a for the A allele, in panel $\mathrm{b}$ for the $\mathrm{G}$ allele, and in panel $\mathrm{c}$ for the heterozygote. Column, monolithic PS/DVB, $50 \times 0.2 \mathrm{~mm}$ i.d.; linear gradient, $50 \%-70 \%$ B in $10 \mathrm{~min}$; flow rate, $3.0 \mu \mathrm{L} / \mathrm{min}$; temperature, $58^{\circ} \mathrm{C}$. 
only (Figure 3, panels a and b), are clearly distinguished from the polymorphic fragments (Figure 3, panel c) by their distinct multiple peaks. However, variations in peak profile are present among the four polymorphic products because they differ in retention behavior as a function of the fluorophore attached. The differences among FAM, HEX, and NED are small enough not to significantly affect the chromatographic profile (i.e., the heterozygote shows four peaks in the chromatogram). In contrast, because of its more hydrophobic nature, the rhodamine dye ROX elutes at a higher acetonitrile concentration than the other three dye-labeled fragments; as a consequence, denaturation sets in at a temperature $2^{\circ} \mathrm{C}$ lower. This may affect the detection sensitivity of multiplex DHPLC experiments for certain mutants for which peaks from heterozygotes only show up in a narrow temperature range (24). One possible solution is to include in the mixture a PCR product with a higher melting tem perature, as determined by the melting algorithm described previously (7). Alternatively, changes in the filter combinations used may allow the use of TET as a fourth fluorophore, which has similar retention characteristics to FAM, HEX, and NED and whose emission maximum is at $545 \mathrm{~nm}$.

In conclusion, fluorescence detection provides a highly specific and sensitive method for polymorphism detection with DHPLC. It has been demonstrated that multiplexed SNP analysis can be achieved with four-color measurements on samples labeled with different dye molecules. Because the same detector can scan up to 25 capillaries, we are in the process of developing a capillary array HPLC system to mimic existing capillary array electrophoresis systems for DNA sequencing (5) and sizing of microsatellites (11), which will result in an approximately 100 -fold increase in throughput over today's commercially available DHPLC systems.

\section{ACKNOWLEDGMENTS}

This work was funded by the National Institutes of Health grant no. 1RO1-HG01932 to P.J.O.

\section{REFERENCES}

1.Cargill, M., D. Altshuler, J. Ireland, P. Sklar, K. Ardlie, N. Patil, N. Shaw, C.R. Lane et al. 1999. Characterization of single-nucleotide polymorphisms in coding regions of human genes. Nat. Genet. 22:231-238.

2.Choy, Y.S., S.L. Dabora, F. Hall, V. Ramesh, Y. Niida, D. Franz, J. Kasprzyk-Obara, M.P. Reeve et al. 1999. Superiority of denaturing high performance liquid chromatography over single-stranded conformation and conformation-sensitive gel electrophoresis for mutation detection in TSC2. Ann. Hum. Genet. 63:383391.

3.Deng, Z., J.H. Morse, S.L. Slager, N. Cuervo, K.J. Moore, G. Venetos, S. Kalachikov, E. Cayanis et al. 2000. Familial primary pulmonary hypertension (gene PPH1) is caused by mutations in the bone morphogenetic protein receptor-II gene. Am. J. Hum. Genet. 67:737-744.

4.Hecker, K.H., P.D. Taylor, and D.T. Gjerde. 1999. Mutation detection by denaturing DNA chromatography using fluorescently labeled polymerase chain reaction products. Anal. Biochem. 272:156-164.

5.Huang, X.C., M.A. Quesada, and R.A. Mathies. 1992. DNA sequencing using capillary array electrophoresis. Anal. Chem. 64: 2149-2154.

6.Huber, C.G., P.J. Oefner, E. Preuss, and G.K. Bonn. 1993. High-resolution liquid chromatography of DNA fragments on highly cross-linked poly(styrene-divinylbenzene) particles. Nucleic Acids Res. 21:1061-1066.

7.Jones, A.C., J. Austin, N. Hansen, B. Hoogendoorn, P.J. Oefner, J.P. Cheadle, and M.C. O'Donovan. 1999. Optimal temperature selection for mutation detection by denaturing HPLC and comparison to single-stranded conformation polymorphism and heteroduplex analysis. Clin. Chem. 45:1133-1140.

8.Kheterpal, I., J.R. Scherer, S.M. Clark, A. Radhakrishnan, J. Ju, C.L. Ginther, G.F. Sensabaugh, and R.A. Mathies. 1996. DNA sequencing using a four-color confocal fluorescence capillary array scanner. Electrophoresis 17:1852-1859.

9.Liu, W., D.I. Smith, K.J. Rechtzigel, S.N. Thibodeau, and C.D. James. 1998. Denaturing high performance liquid chromatography (DHPLC) used in the detection of germline and somatic mutations. Nucleic Acids Res. 26: 1396-1400.

10.Luckey, J.A., H. Drossman, A.J. Kostichka, D.A. Mead, J. D'Cunha, T.B. Norris, and L.M. Smith. 1990. High speed DNA sequencing by capillary electrophoresis. Nucleic Acids Res. 18:4417-4421

11.Mansfield, E.S., M. Vainer, D.W. Harris, P. Gasparini, X. Estivill, S. Surrey, and P. Fortina. 1997. Rapid sizing of polymorphic microsatellite markers by capillary array electrophoresis. J. Chromatogr. A 781:295-305.

12.McCallum, C.M., L. Comai, E.A. Greene, and S. Henikoff. 2000. Targeted screening for induced mutations. Nat. Biotechnol. 18:455457.

13.Molecular Probes. 2000. Handbook of Fluorescent Probes and Research Chemicals, Molecular Probes, Eugene, OR.

14.O'Donovan, M., P.J. Oefner, S.C. Roberts, J.
Austin, B. Hoogendoorn, C. Guy, G. Speight, M. Upadhyaya et al. 1998. Blind analysis of denaturing high-performance liquid chromatography as a tool for mutation detection. Genomics 52:44-49.

15.Oefner, P.J., C.G. Huber, F. Umlauft, G.N. Bert, E. Stimpfl, and G.K. Bonn. 1994. Highresolution liquid chromatography of fluorescent dye-labeled nucleic acids. Anal. Biochem. 223:39-46.

16.Oefner, P.J. and P.A. Underhill. 1998. DNA mutation detection using denaturing high-performance liquid chromatography, p. 7.10.17.10.12. In N.C. Dracopoli, J.L. Haines, B.R. Korf, D.T. Moir, C.C. Morton, C.E. Seidman, J.G. Seidman, D.R. Smith (Eds.), Current Protocols in Human Genetics. Wiley \& Sons, New York.

17.Ophoff, R.A., G.M. Terwindt, M.N. Vergouwe, R. van Eijk, P.J. Oefner, S.M. Hoffman, J.E. Lamerdin, H.W. Mohrenweiser et al. 1996. Familial hemiplegic migraine and episodic ataxia type- 2 are caused by mutations in the $\mathrm{Ca}^{2}+$ channel gene CACNL1A4. Cell 87:543-552.

18.Premstaller, A., H. Oberacher, and C.G. Huber. 2000. High-performance liquid chromatography-electrospray ionization mass spectrom etry of single- and double-stranded nucleic acids using monolithic capillary columns. Anal. Chem. 72:4386-4393.

19.Schriml, L.M., R.J. Peterson, B. Gerrard, and M. Dean. 2000. Use of denaturing HPLC to map human and murine genes and to validate single-nucleotide polymorphisms. BioTechniques 28:740-745.

20.Spiegelman, J.I., M.N. Mindrinos, and P.J. Oefner. 2000. High-accuracy DNA sequence variation screening by DHPLC. BioTechniques 29:1084-1092.

21.Steinmetz, L.M., M. Mindrinos, and P.J. Oefner. 2000. Combining genome sequences and new technologies for dissecting the genetics of complex phenotypes. Trends Plant Sci. 5:397-401.

22.Swerdlow, H. and R. Gesteland. 1990. Capillary gel electrophoresis for rapid, high resolution DNA sequencing. Nucleic Acids Res. 18:1415-1419.

23.Underhill, P.A., L. Jin, A.A. Lin, S.Q. Mehdi, T. Jenkins, D. Vollrath, R.W. Davis, L.L. Cavalli-Sforza et al. 1997. Detection of numerous Y chromosome biallelic polymorphisms by denaturing high-performance liquid chromatography. Genome Res. 7:996-1005.

24.Wagner, T., D. Stoppa-Lyonnet, E. Fleischmann, D. Muhr, S. Pagès, T. Sandberg, V. Caux, R. Moeslinger et al. 1999. Denaturing high-performance liquid chromatography detects reliably BRCA1 and BRCA2 mutations. Genomics 62:369-376.

Received 7 November 2000; accepted 13 December 2000.

\author{
Address correspondence to: \\ Dr. Peter J. Oefner \\ Stanford Genome Technology Center \\ 855 California Avenue \\ Palo Alto, CA 94304, USA \\ e-mail: oefner@genome.stanford.edu
}

